
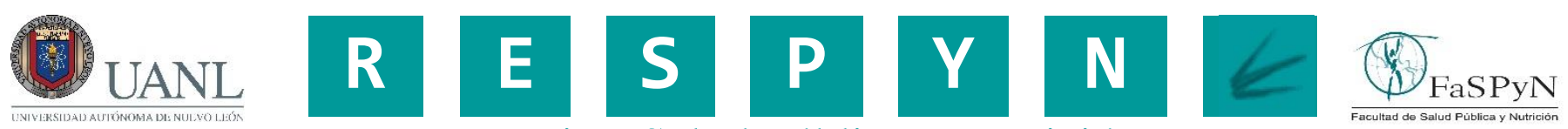

Revista Salud Pública y Nutrición

\title{
PREVALENCIA DE SÍNDROME METABÓLICO EN NIÑOS Y ADOLESCENTES MEXICANOS EN TORNO A SUS DIFERENTES DEFINICIONES.
}

\author{
PREVALENCE OF METABOLIC SYNDROME IN MEXICAN CHILDREN AND ADOLESCENTS IN RELATION TO \\ THEIR DIFFERENT DEFINITIONS.
}

\author{
Ramírez Díaz María del Pilar *, Luna Hernández Jorge Fernando*. \\ *Universidad del Istmo Campus Juchitán, México.
}

\begin{abstract}
Citation: Ramírez Díaz M.P., Luna Hernández J.F. (2019) Prevalencia del síndrome metabólico en niños y adolescentes mexicanos en torno a sus diferentes definiciones. Revista de Salud Pública y Nutrición, 18 (2), 23-32.

Editor: Esteban G. Ramos Peña, Dr. CS., Universidad Autónoma de Nuevo León, Facultad de Salud Pública y Nutrición, Monterrey Nuevo León, México. Copyright: (02019 Ramírez Díaz M.P. et al. This is an open-access article distributed under the terms of Creative Commons Attribution License [CC BY 4.0], which permits unrestricted use, distribution, and reproduction in any medium, provided the original author and source are credited.

Competing interests: The authors have declared that no competing interests exist.

DOI: https://doi.org/10.29105/respyn18.2-4

Recibido: 21 de marzo 2019;

Aceptado: 20 de junio 2019

Email: pilar.ramirezdiaz@gmail.com
\end{abstract}




\title{
PREVALENCIA DE SÍNDROME METABÓLICO EN NIÑOS Y ADOLESCENTES MEXICANOS EN TORNO A SUS DIFERENTES DEFINICIONES
}

\author{
Ramírez Díaz María del Pilar*, Luna Hernández Jorge Fernando*. \\ *Universidad del Istmo Campus Juchitán, México.
}

\section{RESUMEN}

Introducción. El Síndrome Metabólico es factor de riesgo para enfermedad cardiovascular y diabetes tipo 2 . En niños y adolescentes se desconoce la prevalencia real por falta de consenso en sus criterios. Objetivo: Describir la prevalencia de síndrome metabólico en niños y adolescentes mexicanos en torno a sus diferentes criterios diagnósticos. Material y Métodos: revisión descriptiva, de artículos científicos. Enunciados claves: "Síndrome metabólico en niños y adolescentes mexicanos", "Metabolic syndrome in mexican children" y "Criterios diagnósticos de síndrome metabólico en niños mexicanos" utilizando Google Académico, Scielo, Elsevier y Pubmed. Los artículos incluidos fueron los que cumplían con >15 puntos de puntuación STROBE. Resultados: Se encontraron 24 estudios los cuales, registraron 39 prevalencias y 13 criterios diagnósticos. Los más utilizados fueron NCEP-ATPIII, IDF y Cook y las prevalencias mayores fueron 62\%, 54.6\% y 53.3\% con los criterios de Cruz, Cook e IDF en niños con obesidad y las menores $2.0 \%, 2.4 \%$ y $2.6 \%$ con IDF, Weist y Cruz. Conclusiones: El estudio del síndrome metabólico en niños y adolescentes en México debería representar una prioridad para el sistema de salud. Las limitantes del estudio fueron las escasas investigaciones y las diferencias entre prevalencias de acuerdo al criterio utilizado por lo cual, no fue posible establecer una prevalencia certera.

Palabras Clave: Síndrome metabólico en niños y adolescentes, Definición de síndrome metabólico, Criterios diagnósticos de síndrome metabólico.

\section{ABSTRACT}

Introduction: The metabolic syndrome is a risk factor for cardiovascular disease and type 2 diabetes. In children and adolescents, the real prevalence is unknown by the lack of consensus in its diagnostic criteria. Objective: To describe the prevalence of metabolic syndrome in Mexican children and adolescents, around its different diagnostic criteria. Methods: Describe study, literature review. Key phrases used: "Síndrome metabólico en niños y adolescents mexicanos", "Metabolic síndrome in mexican children" and "Diagnostic criterio of metabolic syndrome in mexican children" using Google Académico, Scielo, Elsevier and Pubmed. The studies included were those that met at least 15 STROBE score points. Results: We found 24 studies which registered 39 prevalences and 13 diagnostic criteria. The most used were those of NCEP-ATP III, IDF and Cook. The highest prevalences were $62 \%, 54.6 \%$ and $53.3 \%$ with the criteria of Cruz, Cook and IDF in children with obesity and lowest $2.0 \%, 2.4 \%$ and $2.6 \%$ with IDF, Weist and Cruz. Conclusions: The study of metabolic syndrome in children and adolescents should represent a priority for health system. The limitations of the study were the the scarce investigations and the differences between prevalences according to the criterion used for which, it was not possible to establish an accurate prevalence.

Key words: Metabolic syndrome in children and adolescents, Definition of metabolic syndrome, Diagnostic criteria of metabolic syndrome. 


\section{Introducción}

El Síndrome X o "síndrome de resistencia a la insulina" fue descrito por Reaven (1988) el cual se caracterizaba por diversas alteraciones metabólicas como resistencia a la insulina, obesidad abdominal, hipertensión arterial e inflamación crónica, las cuales aumentaban el riesgo de enfermedad cardiovascular. Años después, en 1998 la Organización Mundial de la Salud (OMS) acuña el término de "síndrome metabólico", cuya definición resalta a la resistencia a la insulina (RI) como uno de los componentes centrales de diagnóstico, requiriendo la implementación del "Clamp Euglucémico Hiperinsulinémico", volviéndola poco práctica y costosa en el ámbito clínico (Alberti y Zimmet,1998). Debido a esto, el Grupo Europeo para el estudio de la Resistencia a la Insulina (EGIR) modificó la definición de la OMS (Balkau,1999) y dos años después, el Programa Nacional de Educación sobre el Colesterol y el Panel III de Tratamiento del Adulto (NCEP-ATP III, 2001) plasma sus criterios diagnósticos de SM, más prácticos, menos invasivos y con mayor aplicación a nivel mundial. Otra de las definiciones más utilizadas es la generada por la Federación Internacional de Diabetes (IDF), la cual señala a la circunferencia abdominal como uno de los principales componentes para el diagnóstico de SM (Alberti y Zimmet, 2005). El SM funge como el principal indicador de riesgo para la generación de diabetes tipo 2 (DT2) y enfermedad cardiovascular (ECV), siendo estas, dos de las principales causas de mortalidad a nivel mundial (NCEP-ATP III, 2001 y OMS, 2018). Dicho síndrome se caracteriza por la presencia de tres o más componentes que incluyen dislipidemias, hipertensión arterial, colesterol HDL disminuido, aumento de la glucemia en ayuno, resaltando a la obesidad abdominal como uno de los componentes más importantes, ya que per se, es un factor de riesgo para la generación de diferentes complicaciones clínico-metabólicas en niños y adultos. (Elizondo et al., 2010; Carrillo et al., 2010 y Asociación Latinoamericana de Diabetes [ALAD], 2013).

Actualmente, la transición demográfica, epidemiológica y nutricional conducen hacia el aumento del consumo de alimentos industrializados y altamente energéticos que ha generado una creciente oleada de casos de sobrepeso y obesidad a nivel mundial propiciando que los casos de SM también se vean aumentados a edades más tempranas. En niños y adolescentes, la prevalencia de obesidad está incrementando de manera descontrolada, tan solo para el año 2016 la OMS estimó que más de 41 millones de niños menores de cinco años en todo el mundo presentaban sobrepeso u obesidad, y a partir de esta edad hasta los 19 años el número de niños y adolescentes obesos se ha multiplicado por 10 en los últimos cuatro decenios, afectando indistintamente a todos los países. (OMS, 2016).

Aunado a lo anterior, el problema de la obesidad y sobrepeso en niños $\mathrm{y}$ adolescentes no pasa desapercibido en México y de acuerdo con los datos de la Encuesta Nacional de Salud y Nutrición de Medio Camino (ENSANUT MC, 2016) la prevalencia de sobrepeso y obesidad para el año 2016 osciló entre el $33.2 \%$ en niños de 5 años a 11 años y $36.3 \%$ en adolescentes de 12 a 19 años (ENSANUT MC, 2016), lo que significa que 1 de cada 3 niños y adolescentes presenta sobrepeso u obesidad, y debido a su magnitud y trascendencia, se ha constituido como un grave problema de salud pública, que sin el manejo adecuado, puede aumentar el riesgo de SM (Vera et al., 2015).Desde esta perspectiva, el diagnóstico de SM en este grupo de edad ha generado controversias por falta de consenso en su definición para niños y adolescentes(Arjona, Gómez y Aguilar, 2008), por lo cual la IDF adapta una clasificación para este grupo poblacional (Zimmet et al., 2007), sin embargo, sigue teniendo límites resolutivos. Esto ha llevado a diversos autores a hacer adaptaciones a los criterios y puntos de corte para el diagnóstico de SM generando que la prevalencia de este problema se vea afectada en función del criterio utilizado.

En nuestro país, el estudio del SM en niños y adolescentes es limitado y se torna difícil establecer la situación en este grupo de edad, por ello, el presente trabajo de investigación tiene como objetivo describir la prevalencia de SM en niños y adolescentes en México en torno a sus diferentes definiciones.

\section{Material y Métodos}

La presente investigación se realizó mediante la revisión de literatura con un enfoque descriptivo y se tomó como muestra principal los artículos científicos que abordaban la descripción y prevalencia del síndrome metabólico en niños y adolescentes 
mexicanos. Se empleó el buscador Google Académico, Scielo, Elsevier y Pubmed para el rastreo de información mediante el uso de los enunciados claves; "Síndrome metabólico en niños y adolescentes mexicanos", "Prevalencia de síndrome metabólico en niños y adolescentes mexicanos", "Metabolic syndrome in mexican children" y "Criterios diagnósticos de síndrome metabólico en niños mexicanos"

Se incluyeron los trabajos que cumplieran las siguientes consideraciones:

1. Investigaciones realizadas en México.

2. Artículos originales.

3. Artículos publicados en cualquier año hasta la actualidad.

4. Artículos publicados en español o inglés.

Los trabajos excluidos fueron aquellos que no fueran originales y los que se repetían en los diferentes motores de búsqueda científica.

Se excluyeron tesis y trabajos que no cumplieran con al menos 15 puntos de la puntuación STROBE.

Para la organización de la información, se fueron vaciando los datos de cada investigación en una tabla incluyendo autor, año, tamaño de muestra del estudio, criterio diagnóstico de síndrome metabólico utilizado y prevalencia obtenida, asegurando que la información fuera lo más homogénea posible.

\section{Resultados}

La búsqueda reflejó que existen pocos estudios referentes al SM en niños y adolescentes en México, y que varios estudios se repiten en las diferentes bibliotecas de artículos científicos.

En total se encontraron 24 artículos que cumplieron con los criterios de inclusión y que describían la prevalencia de SM en niños y adolescentes mexicanos en un periodo que abarca desde el año 2004 al 2018. Se identificó la utilización de 13 criterios diagnósticos diferentes, y los respectivos contrastes entre los componentes y puntos de corte de los mismos.

Como se puede observar (tabla 1) los componentes más utilizados en los criterios diagnósticos fueron los niveles de glucosa, CC, triglicéridos, C-HDL, IMC, PAS, PAD, ICC y la resistencia a la insulina (HOMA-IR). El criterio que mayor número de componentes incluye es el de EGIR con un total de siete, seguido por los criterios de Weist, OMS, y la propuesta cubana con seis. Los criterios del ATP III, IDF, Cook, Ferranti, Cruz y ALAD, comparten cinco componentes (CC, triglicéridos, C-HDL, PA y glucosa) mientras que REGODCI también cuenta con cinco componentes, sin embargo, cambia la CC por el IMC.

De todos los criterios mencionados anteriormente los puntos de corte para CC son menores en el de Ferranti $(\geq \mathrm{P} 75)$ en comparación con los otros cuatro que señalan $\geq P 90$. Este mismo criterio junto con el de la OMS, AACE y Morrison, divide el componente de C-HDL en mujeres y hombres, a diferencia de los demás que lo manejan como un componente general y que los vuelve más específicos.

Los criterios de Weist, EGIR y AACE contemplan los mismos componentes que los criterios anteriormente mencionados, con adición del IMC. Asimismo, el de AACE divide en hombres y mujeres la $\mathrm{CC}$ en $\mathrm{cm}$, mientras que Weist la maneja en percentil >90. Los criterios de IDF, REGODCI, OMS, ALAD, EGIR y AACE, dividen la presión arterial en sistólica y diastólica, mientras que el resto de los criterios maneja un percentil general.

La glucosa, es un componente presente en todos los criterios por su importancia en cuanto al riesgo de diabetes, los puntos de corte de la IDF, REGODCI y ALAD, son los más bajos, (>100 mg/dl) mientras que los otros criterios lo manejan $>110 \mathrm{mg} / \mathrm{dl}$, similares a los de un adulto.

Los puntos de corte de triglicéridos son los que más difieren entre criterios, el de la OMS, ALAD, EGIR, AACE y REGODCI son los más elevados y se clasifican prácticamente como si fueran de un adulto, mientras que los del ATP III y Ferranti son los más bajos.

El criterio de la OMS y la propuesta cubana son los únicos que incluyen el ICC en sus componentes y el único criterio que maneja la resistencia a la insulina (HOMA-IR) es el de EGIR, sin embargo, solo se utilizó en uno de los estudios realizados en niños mexicanos.

Por otro lado, se pueden observar las diferencias entre prevalencias de las investigaciones a través de distintos criterios en la tabla 2; cuatro de los estudios utilizaron criterios diferentes en la misma muestra 

de síndrome metabólico, criterios diagnósticos de síndrome metabólico.

Tabla 1. Criterios diagnósticos de síndrome metabólico en niños y adolescentes

\begin{tabular}{|c|c|c|c|c|c|c|c|c|c|c|c|c|c|}
\hline Parámetros & $\begin{array}{l}\text { NCEP-ATP } \\
\text { III }\end{array}$ & IDF & Cook & Weiss & Ferranti & Cruz & REGOCID & OMS & ALAD & Morrison & EGIR & AACE & $\begin{array}{c}\text { Propuesta } \\
\text { Cubana }\end{array}$ \\
\hline $\begin{array}{l}\text { Circunferencia de } \\
\text { cintura percentil o cm }\end{array}$ & $\geq \mathrm{P} 90$ & $\geq \mathrm{P} 90$ & $>\mathrm{P} 90$ & $>\mathrm{P} 97$ & $>$ P75 & $>\mathrm{P} 90$ & & & $\geq \mathrm{P} 90$ & & $\begin{array}{l}H \geq 94 \mathrm{~cm} \\
M \geq 80 \mathrm{~cm}\end{array}$ & $\begin{array}{l}\mathrm{H}>102 \\
\mathrm{M}>88\end{array}$ & \\
\hline $\begin{array}{l}\text { Triglicéridos Percentil } \\
\text { o mg/dl }\end{array}$ & $\geq 100$ & $\geq 150$ & $\geq 110$ & $>\mathrm{P} 95$ & $\geq 100$ & $>\mathrm{P} 90$ & $\geq 141.6$ & $>150$ & $\geq 150$ & $\geq 110$ & $\geq 180$ & $>150$ & $\begin{array}{l}\mathrm{H}>110 \\
\mathrm{M}>90\end{array}$ \\
\hline $\begin{array}{l}\text { C-HDL Percentil o } \\
\mathrm{mg} / \mathrm{dl}\end{array}$ & $\leq 40$ & $\leq 40$ & $\leq 40$ & $<\mathrm{P} 5$ & $\begin{array}{l}\mathrm{H}<50 \\
\mathrm{M}<44\end{array}$ & $<\mathrm{P} 10$ & $\leq 48.7$ & $\begin{array}{l}\mathrm{H}<40 \\
\mathrm{M}<35\end{array}$ & $\leq 40$ & $\begin{aligned} \mathrm{H} & \leq 40 \\
\mathrm{M} & \leq 50\end{aligned}$ & $\leq 40$ & $\begin{aligned} \mathrm{H} & \leq 40 \\
\mathrm{M} & \leq 50\end{aligned}$ & $<\mathrm{P} 5$ \\
\hline $\begin{array}{l}\text { Presión arterial } \\
\text { Percentil o mm/Hg } \\
\text { PAS y PAD }\end{array}$ & $\geq \mathrm{P} 90$ & $\begin{array}{l}\mathrm{S} \geq 130 \\
\mathrm{D} \geq 85\end{array}$ & $\geq \mathrm{P} 90$ & $>\mathrm{P} 95$ & $\geq \mathrm{P} 90$ & $>\mathrm{P} 90$ & $\begin{array}{l}S \geq 116.5 \\
D \geq 77.1\end{array}$ & $\begin{array}{l}S>140 \\
D>90\end{array}$ & $\begin{array}{l}\mathrm{S} \geq 130 \\
\mathrm{D} \geq 85\end{array}$ & $\geq \mathrm{P} 90$ & $\begin{array}{l}S>140 \\
D>90\end{array}$ & $\begin{array}{l}\mathrm{S}>130 \\
\mathrm{D}>85\end{array}$ & $>95$ \\
\hline Glucosa mg/dl & $\geq 110$ & $\geq 100$ & $\geq 110$ & $\begin{array}{c}>140 \\
<200 \mathrm{PCG}^{\mathrm{a}}\end{array}$ & $>110$ & $\mathrm{TGA}^{\mathrm{b}}$ & $\geq 100$ & $\begin{array}{c}\mathrm{DM}^{\mathrm{c}}, \mathrm{IG}^{\mathrm{d}}, \\
\mathrm{GAA}^{\mathrm{e}}\end{array}$ & $\geq 100$ & $\geq 110$ & $\geq 110$ & $\begin{array}{c}110-125 \text { en } \\
\text { ayuno o } 140- \\
200 \text { PCG }\end{array}$ & $\begin{array}{c}6.1 \mathrm{mmol} / \mathrm{Lo} \\
\text { Glucemia } 2 \\
\text { hrs }>140\end{array}$ \\
\hline IMC & & & & $\begin{array}{c}\text { Z-Score } \geq \\
2.0\end{array}$ & & & $\begin{aligned} H & \geq \mathrm{P} 99 \\
\mathrm{M} & \geq \mathrm{P} 88\end{aligned}$ & $\geq 30$ & & $\geq \mathrm{P} 90$ & $\geq 30$ & $\geq 25$ & $>97$ \\
\hline $\mathrm{C} / \mathrm{C}$ & & & & & & & & $\begin{aligned} \mathrm{H} & >0.90 \\
\mathrm{M} & >0.85\end{aligned}$ & & & & & $>97$ \\
\hline $\begin{array}{l}\text { Resistencia a la } \\
\text { insulina (HOMA-IR) }\end{array}$ & & & & & & & & & & & $>P 75$ & & \\
\hline \multicolumn{14}{|c|}{ Fuente: Elaboración propia } \\
\hline $\begin{array}{l}{ }^{\text {a }} \text { PCG: Post carga } \\
{ }^{\mathrm{b}} \text { TGA: Tolerancia }\end{array}$ & $\begin{array}{l}\text { de glucosa } \\
\text { a a la glucosa a }\end{array}$ & alterada & & $\begin{array}{l}{ }^{\mathrm{e}} \text { DM: } \\
{ }^{\mathrm{d}} \text { IG: In }\end{array}$ & $\begin{array}{l}\text { Diabetes } n \\
\text { tolerancia }\end{array}$ & litus & & ${ }^{\mathrm{e}} \mathrm{GA}$ & : Glucosa & lterada en & & & \\
\hline
\end{tabular}

\begin{tabular}{|c|c|c|c|c|c|c|c|c|c|c|c|c|c|c|c|c|}
\hline \multicolumn{17}{|c|}{ Prevalencias con base a los criterios utilizados (\%) } \\
\hline \multirow[t]{2}{*}{ Primer autor } & \multirow[t]{2}{*}{ Año } & \multicolumn{2}{|c|}{ Población } & \multirow[b]{2}{*}{ NCEP-ATPIII } & \multirow[b]{2}{*}{ IDF } & \multirow[b]{2}{*}{ Cook } & \multirow[b]{2}{*}{ Weiss } & \multirow[b]{2}{*}{ Ferranti } & \multirow[b]{2}{*}{ Cruz } & \multirow[b]{2}{*}{ REGODCI } & \multirow[b]{2}{*}{ OMS } & \multirow[b]{2}{*}{ ALAD } & \multirow[b]{2}{*}{ Morrison } & \multirow[b]{2}{*}{ EGIR } & \multirow[b]{2}{*}{ AACE } & \multirow[b]{2}{*}{ Cuba } \\
\hline & & Muestra & Edad (años) & & & & & & & & & & & & & \\
\hline Rodríguez M. & 2004 & 965 & 10 a 18 & 6.5 & - & - & - & - & - & 7.8 & 4.5 & - & - & 3.8 & 7.7 & - \\
\hline Halley E. & 2007 & 1366 & $7 a 24$ & 19.6 & - & - & - & - & - & - & - & - & - & - & - & - \\
\hline Aradillas $\mathrm{G}$. & 2008 & 871 & 6 a 15 & - & - & - & - & - & - & 26.6 & - & - & - & - & - & - \\
\hline Jiménez A. & 2009 & 246 & 12 a 14 & - & - & - & - & - & - & - & - & - & 16 & - & - & - \\
\hline Guerrero F. & 2010 & 1262 & 7a 15 & - & - & 9.2 & - & - & - & - & - & - & - & - & - & - \\
\hline Martínez J. & 2010 & 97 & 3а 18 & 37.1 & - & - & - & - & - & - & - & - & - & - & - & - \\
\hline Cárdenas V. & 2010 & 254 & 10 a 19 & 9.4 & - & - & - & - & - & - & - & - & - & - & - & - \\
\hline Cardoso G. & 2010 & 1850 & 12 a 16 & 12.6 & - & - & - & - & - & - & - & - & - & - & - & - \\
\hline Bautista L. & 2010 & 150 & 6 a 16 & - & - & - & - & - & - & - & - & - & - & - & - & 33.3 \\
\hline Elizondo L.* & 2010 & 215 & 6 a 12 & - & 6.7 & - & - & - & - & - & - & - & - & - & - & - \\
\hline Elizondo L.* & 2011 & 261 & 6 a 12 & - & - & 23.3 & - & - & - & - & - & - & - & - & - & - \\
\hline Evia M.** & 2013 & 110 & 8 a 16 & - & - & - & - & - & 62 & - & - & - & - & - & - & - \\
\hline Elizondo L.* & 2013 & 96 & 6 a 12 & - & - & 44 & - & - & - & - & - & - & - & - & - & - \\
\hline Álvarez M. & 2014 & 972 & 16 a 20 & - & 4.63 & - & - & - & - & - & - & - & - & - & - & - \\
\hline Ortega R. & 2014 & 103 & 10 a 15 & - & 53.3 & - & - & - & - & - & - & - & - & - & - & - \\
\hline Ortega R. & 2015 & 172 & 6 a 15 & - & - & - & - & - & - & - & - & 48.8 & - & - & - & - \\
\hline Romero $\mathrm{E}$. & 2016 & 120 & 8 a 13 & 48.6 & 33.3 & - & - & - & - & - & 40.3 & - & - & - & - & - \\
\hline Peña B. & 2017 & 508 & 9 a 13 & 21.1 & 15.4 & 13.8 & 2.4 & 45.9 & - & - & - & - & - & - & - & - \\
\hline Valdés Y. & 2017 & 2599 & 6 a 16 & 5.1 & 2 & 4.8 & - & 12.9 & 2.6 & 26.6 & - & - & - & - & - & - \\
\hline Gonzáles C. & 2018 & 60 & 4a 17 & - & - & 43 & - & - & - & - & - & - & - & - & - & - \\
\hline Ávila A.* & 2018 & 1017 & 6 a 12 & - & - & 54.6 & - & - & - & - & - & - & - & - & - & - \\
\hline
\end{tabular}

con el objetivo de hacer comparaciones de prevalencias; identificando un total de 39 prevalencias distintas entre todas las investigaciones. También se muestran los criterios utilizados en cada una de las prevalencias obtenidas, el criterio más utilizado por los diferentes autores fue el de NCEPATPIII con un porcentaje de $23.07 \%$ (9), continuando por el propuesto por la IDF con el 20.51 $\%$ (8), seguido por la propuesta de Cook con $17.94 \%$ (7). En contraste, los criterios menos utilizados fueron los de Weist, ALAD, EGIR, Morrison y la propuesta cubana con un $4.1 \%$ (1).
El menor tamaño de muestra utilizada por los autores fue de 60 , mientras que la mayor estuvo constituida por 2599 individuos, el rango de edad de los sujetos de estudio estuvo entre tres y 24 años. De estas muestras, el $50 \%$ (12) estuvieron constituidas por niños y adolescentes sin distinción de su IMC. Por otro lado, $16.6 \%$ (4) fueron constituidas por niños y adolescentes con sobrepeso y obesidad exclusivamente, $20.8 \%$ (5) de los estudios fueron realizados en población con obesidad, $8.3 \%$ (2) en población con obesidad y normopeso y el $4.1 \%$ (1) estuvo constituida por niños y adolescentes con obesidad, sobrepeso y normopeso. 
Las tres prevalencias más altas registradas fueron obtenidas con los criterios de Cruz, Cook e IDF siendo estas de $62 \%, 54.6 \%$ y $53.3 \%$ respectivamente. Los tres criterios diagnósticos manejan los mismos indicadores, sin embargo, los puntos de corte guardan ciertas diferencias. Es importante mencionar que la muestra de dichos estudios estaba integrada exclusivamente por niños y adolescentes con presencia de obesidad. En contraste, las prevalencias menores fueron obtenidas en aquellos estudios que utilizaron los criterios de IDF $(2.0 \%)$, Weist (2.4\%) y Cruz (2.6\%), teniendo en cuenta que en la muestra de estudio no se consideró el IMC como criterio de selección.

Dentro de los cuatro estudios que utilizaron más de un criterio diagnóstico para comparar las prevalencias de SM dentro de una misma muestra se encontraron diferencias desde $2.4 \%$ al $45.9 \%$, lo que refleja las discrepancias entre diferentes criterios diagnósticos en una misma población.

\section{Discusión}

En México, el panorama epidemiológico de las enfermedades crónicas no transmisibles (ECNT) es grave, de acuerdo con cifras de la ENSANUT MC (2016) la prevalencia conjunta de sobrepeso y obesidad en población mayor a 20 años es de alrededor del $72.5 \%$ y $9.4 \%$ para diabetes tipo 2 , cifra que podría incrementarse al doble si se admite el criterio de que por cada persona diagnosticada existe otra que desconoce padecerla.

En cuanto a niños y adolescentes la situación no mejora, la problemática se ha visto exacerbada por el cambio en los estilos de vida, el sedentarismo, la transición epidemiológica y nutricional dirigida hacia el consumo de alimentos altamente energéticos, con alto contenido de azúcar añadida, grasas saturadas, sodio y la incorrecta aplicación de las políticas públicas para el control de la industria alimentaria. Debido a esto, la prevalencia de sobrepeso y obesidad para el año 2016 osciló entre el $33.2 \%$ en niños de 5 años a 11 años y $36.3 \%$ en adolescentes de 12 a 19 años (ENSANUT MC, 2016), lo que significa que 1 de cada 3 niños y adolescentes presenta sobrepeso $\mathrm{u}$ obesidad, incrementando su probabilidad a desarrollar complicaciones metabólicas.
El vínculo entre la obesidad, SM y DT2 ya se ha caracterizado en poblaciones adultas y se ha documentado que las personas con SM tienen el doble de probabilidades de morir y tres veces más probabilidades de tener complicaciones cardiovasculares (Zimmet et al., 2007). En población pediátrica y adolescente se ha tornado más complicado su estudio, siendo uno de los principales problemas la existencia de una amplia variedad de criterios que utilizan diferentes componentes, los cuales, incurren en las prevalencias. La construcción de una definición de SM para niños y adolescentes es compleja, ya que existen diferentes cambios metabólicos relacionados con el desarrollo puberal como la presión arterial, los niveles de lípidos y la composición corporal que afectan la distribución del tejido adiposo y provocan una disminución en la sensibilidad a la insulina de hasta el $30 \%$, con un consecuente aumento en la secreción de la misma (Alberti G. et al., 2004);así como de los niveles de adiponectina (Reinehr, Roth, Menke y Andler, 2004), a consecuencia de esto diversos autores han optado por hacer adaptaciones a las definiciones ya establecidas para adultos, como el criterio de ATPIII; sin embargo dichas adaptaciones se hicieron sobre la base de patrones de crecimiento y desarrollo de los Estados Unidos, lo que complica la aplicación universal para definir SM en niños y adolescentes.

Es importante mencionar que cada una de las definiciones están creadas con base a una muestra poblacional distinta, por lo cual, se tendría que elegir aquellas definiciones que ocupen muestras representativas con características similares a la población que se estudiará, en este sentido, una definición que podría ser útil para población mexicana sería la de la Asociación Latinoamericana de Diabetes (ALAD,2007), ya que los puntos de corte están establecidos para población latinoamericana de 6 a 18 años, sin embargo, solo una de las investigaciones encontradas hizo uso de esta definición y no hay manera de comparar resultados, por ello sería necesario seguir evaluando su aplicación en nuevos estudios en paralelo con otras definiciones y así observar las diferencias en las prevalencias obtenidas.

De manera general la revisión muestra una diferencia evidente entre las prevalencias de SM en niños y adolescentes, esto a consecuencia de los distintos criterios utilizados, además del tipo de muestra 
seleccionada para su estudio, debido a que, las prevalencias más altas fueron encontradas en niños y adolescentes con presencia de sobrepeso, obesidad o ambas. Coincidiendo con la fisiopatología propia de la obesidad, ya que la presencia de esta condición propicia alteraciones clínicas y metabólicas como hipertensión, hiperglucemia y dislipidemias, representando un predictor de enfermedades cardiovasculares (ECV) y diabetes tipo 2 (DM2). (NCEP-ATP III, 2001; Weiss y Caprio, 2005; Mamum et al., 2009; Wong, 2007 y Kurl S et al., 2006). Estudios han determinado prevalencias del $30 \%$ de SM en población infantil con obesidad (Cook, 2004) y hasta un 50\% con mayor grado de obesidad (Weiss et al., 2004); En consecuencia, se podría esperar una prevalencia considerable en nuestro país ya que, de acuerdo a lo reportado por la ENSANUT MC las cifras de prevalencia de obesidad en niños de 5-11 fue de 15.3\%, mientras que para los adolescentes de 12-19 años fue de $13.9 \%$.

Ante esta situación, sería importante reflexionar el uso correcto de un mismo criterio para el diagnóstico de SM en población pediátrica obesa y no obesa, por ello, se podría pensar en utilizar puntos de corte más estrictos en niños con obesidad ya que, probablemente algunos de estos casos aún no presenten cambios metabólicos debido al tiempo de evolución de su condición, sin embargo, esto no significa que se encuentren fuera de riesgo de presentar en un futuro SM o en su defecto otra enfermedad metabólica, por lo cual, su vigilancia tendría que ser más rigurosa por el personal de salud. Por otro lado, existen otros componentes clínicos y metabólicos relacionados con el SM que podrían ahondar en su diagnóstico como lo es la hiperuricemia, ya que esta relación va acorde con la fisiopatología, al tener una alteración de la insulinemia aumentan los niveles de ácido úrico. (Denzer et al., 2003 y Ford et al., 2007; Tsouli et al., 2006; Sui et al., 2008; Mangge et al.,2013 y Cardoso et al., 2013). En el mismo sentido se han asociado biomarcadores alterados como la proteína $\mathrm{C}$ reactiva y los niveles de interleucinas-6 propios de la inflamación como consecuencia de la RI y la obesidad. (Weiss et al., 2004) De igual modo se han relacionado las concentraciones alteradas de adiponectinas almacenadas en el tejido adiposo blanco, como una característica presente en la obesidad, SM, incluso en la enfermedad hepática grasa no alcohólica, la cual también se ha considerado como un componente adicional al SM, debido a que representa un signo prematuro de enfermedades cardiovasculares en adultos y niños. (Kotnik, Fischer y Wabitsch, 2015; Alterio et al., 2014 y Bussler et al., 2017)

Existen indicadores en cuanto a costo- beneficio que han demostrado su efectividad en otros componentes del SM, un ejemplo ya conocido es la medición de la circunferencia de cintura, ésta ha demostrado ser un mejor indicador de riesgo de hipertensión e hipertrigliceridemia, con respecto al IMC. Por lo que se ha considerado que el IMC sobre los factores de riesgo cardiovascular, se encuentra influenciado por la obesidad abdominal. (Balas et al., 2008)

En el mismo sentido existen un indicador que recientemente han tomado mayor auge dentro de la investigación como es el caso del índice cintura talla (ICT) el cual, ha demostrado en algunos estudios tener mayor fuerza de asociación con el riesgo de enfermedades cardiovasculares (ECV) en comparación al IMC (Elizondo AshwellyHsieh, 2005; Kahn, Imperatore y Cheng, 2005;Maffeis, Bazanto y Talamini, 2008; Elizondo, 2011),en el mismo sentido los resultados de un metaanálisis demostraron que este indicador fue un mejor predictor de manera general para determinar factores de riesgo de enfermedades cardio metabólicas en niños; además, de que tiene la bondad de no necesitar puntos de corte específicos para la edad, sexo o grupo étnico, por lo cual facilita su interpretación. (Lo. k, Wong, Khalechelvam y Tam , 2016).

Ante el panorama ya mencionado, es imprescindible realizar un tamizaje oportuno desde el primer nivel de atención, no obstante, en la práctica clínica no se realiza de manera efectiva, tal como lo muestra un estudio realizado por el Instituto Nacional de Ciencias Médicas y Nutrición Salvador Zubirán (Medina, 2015) cuyo objetivo fue explorar la capacidad de los profesionales dela salud para identificar los principales criterios de SM en niños en dos estados de la república. En dicho estudio se observó que el $63.1 \%$ del total de los profesionales no miden la circunferencia de cintura en preescolares, siendo uno de los principales factores de riesgo para desarrollar SM, el $46.4 \%$ no la medía en escolares, sin embargo, el porcentaje aumentó en adolescentes hasta el $64.6 \%$. Por otro lado, se reportó que el $72.6 \%$ de los encuestados no mide la presión arterial en edad 
preescolar y el $64.6 \%$ en edad escolar. A pesar de que el estudio solo describe la situación de dos estados de la república, podría estar reflejando el desconocimiento o la falta de una búsqueda intencionada de los factores de riesgo de SM en pacientes pediátricos por parte del personal de salud, una de las razones del porque no se realiza en la consulta clínica, puede deberse a que dichos factores se hacen presentes en mayor proporción en personas de edades más avanzadas, no obstante, ante la magnitud que representa actualmente la obesidad infantil es indispensable incluir indicadores que nos permitan tener una detección temprana en niños y adolescentes que estén en riesgo o ya presenten SM. Dentro de las limitantes que presenta esta revisión encontramos que existen escasos estudios acerca de la prevalencia de SM en niños y adolescentes mexicanos y en su mayoría utilizan criterios y muestras distintas que no nos permiten hacer comparaciones homogéneas, ni dar una cifra certera acerca de la prevalencia en México.

\section{Conclusiones}

El estudio de SM en niños y adolescentes en México debería representar una prioridad para el sistema de salud, debido a la alta prevalencia de obesidad infantil cuyas complicaciones se hacen presentes en edades más tempranas. La estandarización de criterios y puntos de corte han representado una gran limitante dentro de la investigación, es por ello, que surge la necesidad de elaborar criterios propios, adaptados a las condiciones y características de población mexicana. Dichos criterios contribuirían a dar un panorama más certero acerca de la prevalencia de SM, lo cual, favorecería a la toma de decisiones en cuanto a la vigilancia epidemiológica y a las posibles intervenciones que favorezcan el control y la disminución de complicaciones de casos. Por otro lado, es importante resaltar la relevancia de capacitar al personal de salud acerca de incluir nuevas mediciones factibles y de fácil interpretación durante la práctica clínica, que nos permita indagar a mayor profundidad sobre el estado o la condición en la que se encuentre tanto el infante como el adolescente, con el objetivo de detectar posibles casos de SM y disminuir la prevalencia de ECNT.

\section{Bibliografía}

Alberti K., Zimmet P., Shaw J., BloomgardenZ.,Kaufman F. y Silink M. (2004). Type 2 Diabetes in the Young: the evolving epidemic. Diabetes Care, 27(7), 17981811. doi:10.2337/diacare.27.7.1798
Alberti K., Zimmet P. (1998). Definition, Diagnosis and Classification of Diabetes Mellitus and its Complications Part 1. Diagnosis and classificatión of Diabetes Mellitus Provisional Report of a WHO Consultation. Diabet Med, 15, 539-53. doi: /10.1002/(SICI)1096-9136(199807)15:7<539::AIDDIA668>3.0.CO;2-S

Alberti K., Zimmet P. (2005). Una nueva definición mundial del síndrome metabólico propuesta por la Federación Internacional de Diabetes : fundamento y resultados.RevEspCardiol. 58(12): 1371-6. doi: 10.1016/S0300-8932(05)74065-3

Álvarez M., Hernández MR., Jiménez M. y Durán A. (2014). Estilo de vida y presencia de síndrome metabólico en estudiantes universitarios. Diferencias por sexo. Rev Psicol, 32(1), 123-138. Recuperado de: http://www.scielo.org.pe/pdf/psico/v32n1/a05v32n1.p $\mathrm{df}$

American Association of Clinical Endocrinologists (AACE). (2003). Position statement on the insulin resistance Syndrome. EndocrinePractice, 9(2),5-21. Recuperado de: https://www.aace.com/files/americancollege-of-endocrinology-position-statement-on-theinsulin-resistance-syndrome.pdf

Arjona R., Gomez R., y Aguilar A. Controversias en el diagnóstico del síndrome metabólico en poblaciones pediátricas. Bol MedHospInfantMex, 65, 488-501. Recuperado http://www.medigraphic.com/pdfs/bmhim/hi2008/hi086h.pdf

Ashwell M. y Hsieh SD. (2005). Six reasons why the waistto-height ratio is a rapid and effective global indicator for health risks of obesity and how its use could simplify the international public health message on obesity. Int J FoodSciNutr, 56(5), 303-307. doi: /10.1080/09637480500195066

Asociación Latinoamericana de Diabetes. (2013). Diagnóstico, control y tratamiento del Síndrome Metabólico en Pediatría. ALAD, 17(1), 16-31. Recuperado de:http://www.revistaalad.com/pdfs/0905_Guia_ALA D.pdf

Ávila A., Galindo C., Juárez L. y Osorio M. Síndrome metabólico en niños de 6 a 12 años con obesidad, en escuelas públicas de siete municipios del Estado de México. SaludpúblicaMéx 60(4), 395-403. doi.org/10.21149/8470 
Balas M., Villanueva A., Tawil S., Shiffman E., Suverza A., Vadillo F. y Perichart Otilia. (2008). Estudio piloto para la identificación de indicadores antropométricos asociados a marcadores de riesgo de síndrome metabólico en escolares mexicanos. Bol Med Hosp Infant Mex, 68 (2), 100-109. Recuperado de: https://www.medigraphic.com/pdfs/bmhim/hi2008/hi082d.pdf

Balkau B. (1999). Comment on the provisional report from the WHO consultation. European Group for the Study of Insulin Resistance (EGIR). Diabet Med, 16, 442-443 doi: 10.1046/j.1464-5491.1999.00059.x

Bautista L., Saldaña M. y Hernández JC. (2010). Síndrome metabólico en población de 6 a 16 años de edad en primer nivel de atención. Revista de la Facultad de Medicina de la UNAM, 53(4), 3-8. Recuperado de: http://www.pve.unam.mx/informacion/medicina/RFM JULIO.pdf\#page $=3$

Bussler S., Penke M., Flemming G., Elhassan Y.S., Kratzsch J., Sergeyev E., Lipek T., Vogel M., Spielau U., Körner A. de Giorgis T. y Kiess W.(2017). Nocel insights in the metabolic síndrome in childhood and adolescence. Horm Res Paediatr, 88, 181-193. doi: $10.1159 / 000479510$

Calderín O., Prieto M. y Cabrera E. (2007). Síndrome de insulinorresistencia en niños y adolescentes. RevCubanaEndocrinol, 18(2). Recuperado de: http://scielo.sld.cu/scielo.php?script=sci_arttext\&pid= S1561-29532007000200007

Camarillo R., García M., Amaya C.,Huitrón B y Majluf C. (2010). Dificultades en la clasificación del síndrome metabólico. El ejemplo de los Adolescentes en México. Salud Pública Mex, 52(6), 524-27. Recuperado de: http://www.medigraphic.com/pdfs/salpubmex/sal2010/sal106g.pdf

Cárdenas V., López JC., Bastarrachea R., Rizo M. y Cortés E. (2010). Prevalencia de síndrome metabólico y sus componentes en adolescentes de la Ciudad de Monterrey, Nuevo León. ArchCardiolMéx, 80(1), 19$26 . \quad$ Recuperado de: http://www.scielo.org.mx/pdf/acm/v80n1/v80n1a5.pd f

Cardoso AS, Gonzaga NC, Medeiros CCM, de Carvalho DF. (2013) Association of uric acid levels with components of metabolic syndrome and non-alcoholic fatty liver disease in overweight or obese children and adolescents. J Pediatr (Rio J), 89(84), 412-418. doi: 10.1016/j.jpedp.2012.12.011
Cardoso G., Yamamoto L., Medina A., Posadas R., Caracas N. y Posadas Carlos. (2010). Exceso de peso y síndrome metabólico en adolescentes de la Ciudad de México. ArchCardiolMéx, 80(1), 12-18. Recuperado de:

http://www.scielo.org.mx/pdf/acm/v80n1/v80n1a4.pd f

Cook S., Weitzman M., AuingerP.,et al.(2003). Prevalence of a metabolic síndrome phenotype in adolescents. Arch PediatrAdolesc Med, 157(8), 821-27. doi:10.1001/archpedi.157.8.821

Cook S. (2004). The metabolic syndrome: Antecedent of adult cardiovascular disease in pediatrics. $J$ Pediatr.145(4), 427-30. doi: 10.1016/j.jpeds.2004.07.021

Cruz M., Weigensberg M., Huang T., Ball G., Shaibi G. y Goran M. (2004). The metabolic síndrome in overweight hispanicyought and the role of insulin sensitivity. J ClinEndocrinolMetab, 89(1), 108-113. doi: $10.1210 /$ jc.2003-031188

Denzer C, Muche R, Mayer H, Heinze E, Debatin KM, Wabitsch M. (2003). Serum uric acid levels in obese children and adolescents: Linkage to testosterone levels and pre-metabolic syndrome. $J$ Pediatr Endocrinol Metab, 16(9), 1225-32. doi: 10.1515/JPEM.2003.16.9.1225

Elizondo L., Gutiérrez N., Moreno D., Martínez U., Tamargo D. y Treviño M. (2013). School-based individualised lifestyle intervention decreases obesity and the metabolic síndrome in mexican children. $J$ Hum Nutr Diet, 26, 82-89. doi:10.1111/jhn.12070

Elizondo L., Serrano G., Ugalde C., Cuello G. y Borbolla E. (2010) Metabolic síndrome risk factors among a sample of overweight and obese Mexican children. $J$ ClinHypertens, 12(5), 380-87. doi: 10.1111/j.17517176.2010.00263.x

Elizondo L., Serrano M., Ugalde P., Bustamante H. y Cuello C. (2011). Waist-height: cutoff matters in predicting metabolic síndrome in mexican children. Metab Syndr Relat Disord, 9(2), 183-190. doi: 10.1089/met.2010.0116

Encuesta Nacional de Salud y Nutrición de Medio Camino. (2016). Informe final de resultados. Recuperado de: https://www.gob.mx/cms/uploads/attachment/file/ 209093/ENSANUT.pdf

Evia M., Rodea E.,Apolinar E y Quintana S. (2013). Metabolic síndrome and its components among obese 
(BMI $\geq 95$ th) mexican adolescents. Endocr Connect, 2(4), 208-215. doi: 10.1530/EC-13-0057

Ferranti S., Gauvreau K., Ludwig D., Neufeld E., Newburger J. y Rifai N. (2004). Prevalence of the Metabolic Syndrome in American Adolescents. Circulation, 110(16), 2494-97. doi: 10.1161/01.CIR.0000145117.40114.C7

Ford ES., Li C., Cook S. y Choi HK. (2007) Serum concentrations of uric acid and the metabolic syndrome among US children and adolescents. Circulation. 115(19), 2526-32. doi: 10.1161/CIRCULATIONAHA.106.657627

González C., Guevara E., Gutiérrez P., Tlacuilo J., Sánchez M., García A. y Barba JF. (2018). Síndrome metabólico y aterosclerosis carotídea subclínica en niños y adolescentes mexicanos con acantosis negricans. GacMedMex, 154(4), 462-67. doi://dx.doi.org/10.24875/GMM.18003699

Guerrero F., Aradillas C., Simental L., Monreal E.,Cruz E. y Rodríguez M. (2010). Birth weight, family history of diabetes, and metabolic syndrome in children and adolescents. The Journal of Pediatrics, 156(5), 719-23. doi: 10.1016/j.jpeds.2009.11.043

Guzmán I., Salgado A., Muñoz J., Vences A. y Parra I. (2013) Prevalencia de síndrome metabólico en niños con obesidad y sin ella. Med Clin (Barc), 144(5) 198203.doi: 10.1016/j.medcli.2013.10.033

Halley E., Borges G., Talavera J., Orozco R., Vargas C., Huitrón G., Diaz JC., Castañón S ySalmerón J. (2007). Body mass index and the prevalence of metabolic syndromeon children and adolescents in two mexican populations. Journal of Adolescents Health, 40(6), 521-26. doi: 10.1016/j.jadohealth.2006.12.015

Jiménez A., Velasco RM., Bacardí M., Higuera DF. y Domínguez PE. (2009). HOMA-IR, síndrome metabólico y hábitos dietéticos en adolescentes de Chiapas, México. RevBiomed, 20(2), 82-88. Recuperado de: http://www.medigraphic.com/pdfs/revbio/bio2009/bio092c.pdf

Kahn H, Imperatore G, Cheng Y.(2005). A populationbased comparison of BMI percentiles and waist-toheight ratio for identifying cardiovascular risk in youth. J Pediatr, 146(4), 482-488. doi: 10.1016/j.jpeds.2004.12.028

Klunder M., Flores S., García R., Peralta J. y Cruz M. (2013). Adiponectin in eutrophic and obesechildren as a biomarkertopredictmetabolic síndrome and eachofitscomponents. BMCPublicHealth, 13 (88). doi:10.1186/1471-2458-13-88

Kotnik P., Fischer PP. y Wabitsch M. (2015). Endocrine and metabolic effects of adipose tissue in children and adolescents. Zdr Varst, 54(2),131- 138. doi 10.1515/sjph-2015-0020

Kurl S., Laukkanen J., Niskanen L., Laaksonen D., Sivenius J., Nyyssonen K. y Salonen J. ( 2006). Metabolic syndrome and the risk of stroke in middleaged men. Stroke, 37, 806-11. doi: 10.1161/01.STR.0000204354.06965.44

Lo K., Wong., Khalechelvam P. y Tam W. (2016). Waistto-height ratio, body mass index and waist circumference for screening pediatric cardio-metabolic risk factors: a meta-analysis. ObesityReviews, 17(12), 1258-1275. doi: 10.1111/obr.12456

Maffeis C., Bazanto C. y Talamini G. (2008). Waist to height ratio, a useful index to identify high metabolic risk in overweight children. Pediatr, 152, 207-213. doi: 10.1016/j.jpeds.2007.09.021

Mamum A., Cramb S., O’Callaghan M., Williams G. y Najman J. (2009). Childhood overweight status predicts diabetes at age 21 years: A follow-up study. Obesity, 17(6), 1255-61. doi: 10.1038/oby.2008.660

Mangge H, Zelzer S, Puerstner P, et al. (2013). Uric acid best predicts metabolically unhealthy obesity with increased cardiovascular risk in youth and adults. Obesity (Silver Spring), 21(1),71-77. doi: 10.1002/oby.20061

Martínez J., Rojas P. y León S. (2010). Prevalencia de resistencia a la insulina y síndrome metabólico en niños obesos que acuden a la clínica de obesidad del hospital pediátrico de Sinaloa. PediatrMex, 12(1), 1822. Recuperado de: http://www.medigraphic.com/pdfs/conapeme/pm2010/pm101d.pdf

McCarthy HD y Ashwell M.(2006). A study of central fatness using waist-to-height ratios in UK children and adolescents over two decades supports the simple message-keep your waist circumference to less than half your height. Int $J$ Obes, 30,988-992. doi: 10.1038/sj.ijo.0803226

Medina I., Serralde A., Islas L., Jiménez E., Jiménez K. y Guevara M. (2015). Diagnosis of metabolic syndrome in children as a potential indicator of technical ability in medical and nutritional care.NutrHosp, 32(5),20152110. doi:10.3305/nh.2015.32.5.9703 
Morrison J., Aronson L., y Gray-McGuire C. (2007). Metabolic syndrome in childhood predicts adult cardiovascular disease 25 years later: the priceton lipid research clinics follow-up study. Pediatrics, 120(2), 340-345. doi: 10.1542/peds.2006-1699

Nacional Cholesterol Education Program. (2001). Executive Summary of the Third Report of the National Cholesterol Education Program (NCEP) Expert Panel on Detection. JAMA, 285 (19),248697.doi:10.1001/jama.285.19.2486

Organización Mundial de la Salud. (2016). Informe de la Comisión para acabar con la obesidad infantil. Biblioteca de la OMS. Recuperado de: https://www.who.int/end-childhood-obesity/facts/es/

Ortega R., Hurtado EF., López AL., Trujillo X., Tlacuilo J. y Colunga C. (2014). Caracterización de niños obesos con y sin diagnóstico de síndrome metabólico en un hospital pediátrico. Rev Med Inst Mex Seguro Soc, 52(1), 548-556. Recuperado de: http://www.medigraphic.com/pdfs/imss/im2014/ims141i.pdf

Ortega R., Trujillo X., Hurtado EF., López AL., Colunga C., Barrera JC., y Tlacuilo J. (2015). Componentes clásicos y no tradicionales del síndrome metabólico en niños y adolescentes con exceso ponderal. Nutr Clin Diet Hosp, 35(2), 57.66. doi: 10.12873/352ortega

Peña B., Granados M., Sánchez K., Ortiz G. y Menjivar M. (2017). Síndrome metabólico en niños mexicanos: poca efectividad de las definiciones diagnósticas. Endocrinol Diabetes y Nutr, 64(7), 369-376. doi: 10.1016/j.endinu.2017.04.004

Reaven GGM. (1988). Role of Insulinresistance in human disease. Nutrition, 13(1),64-66. Recuperado de: https://pdfs.semanticscholar.org/3288/2b 39180d12d79bc5a2d204d5b65dba7ddb1e.pdf

Reinerhr T., Roth C., Menke T. y Andler W. (2004). Adiponectin before and after weight los in obese children. J ClinEndocrinolMetab, 89(8), 3790-94. doi: 10.1210/jc.2003-031925

Rodríguez M., Salazar B., Violante R. y Guerrero F. (2004). REGODCI. Metabolic Syndrome Among Children and Adolescents Aged 10-18 years. Diabetes Care, 27(10), 2516-2517. doi: 10.2337/diacare.27.10.2516

Romero E., Aguirre L., Álvarez Y., Vásquez E., Casillas E. y Fonseca S. (2016). Prevalencia de síndrome metabólico y factores asociados en niños y adolescentes con obesidad. RevMedInstMex Seguro
Social, 54(5). 568-575. Recuperado de: http://www.medigraphic.com/pdfs/imss/im2016/im165d.pdf

Sui X, Church TS, Meriwether RA, et al.(2008). Uric acid and the development of metabolic syndrome in women and men. Metabolism, 57(6), 845-52. Doi: 10.1016/j.metabol.2008.01.030

Tsouli SG, Liberopoulos EN, Mikhailidis DP, et al. (2006). Elevated serum uric acid levels in metabolic syndrome: An active component or an innocent by stander. Metabolism, $55(10), 1293$ 301.doi:10.1016/j.metabol.2006.05.013

Valdéz Y., Campuzano J., Sánchez L., Bermudez V., Peralta O., Díaz C., Cruz M. y Burguete A. (2018). Estudio de validación de cuatro diferentes criterios para el diagnóstico de síndrome metabólico en población infantil. Rev Univ Ind Santander, 50(2), 126135. doi: 10.18273/revsal.v50n2-2018004

Vera I., Zuñiga A., Ortega L. Rolland E., Chanes K. y Cruz M. (2015). Diagnosis of metabolic syndrome in children as a potential indicator of technical ability in medical and nutritional care. Nutr Hosp, 32(5):21052110. doi: 10.3305/nh.2015.32.5.9703

Villamor E., Finan C., Ramírez M. y Roman A. (2016). Prevalence and sociodemographic correlates of metabolic syndrome in school-aged children and their parents in nine Mesoamerican countries. Public Health Nutrition. doi:10.1017/S1368980016002342

Weiss R., Dziura J., Burgert T., Tamborlane W., Taksali S., YeckelC.,...Caprio S. (2004). Obesity and the metabolic syndrome in children and adolescent. NEngl $J$ Med, 350, 2362-2374. doi: 10.1056/NEJMoa031049

Weiss R. y Caprio S. (2005). The metabolic consequences of childhood obesity. Best Pract Res Clin Endocrinol Metab.19(3), 405-19. doi: 10.1016/j.beem.2005.04.009

Wong N. (2007). Metabolic syndrome: Cardiovascular risk assessment and management. Am J Cardiovasc Drugs, 7(4), 259-72. doi: 10.2165/00129784-20070704000004

Zimmet P., Alberti K., Kaufman F., Tajima N., Silink M., ArslanianS.,...Caprio S. (2007). The metabolic syndrome in children and adolescents-an IDF consensus report.Pediatric Diabetes, 8, 299-306. doi: 10.1111/j.1399-5448.2007.00271.x 